\title{
ANALIZANDO LA INFLUENCIA DE LA EDUCACIÓN UNIVERSITARIA EN EL PERFIL EMPRENDEDOR DE LOS ESTUDIANTES 1
}

\author{
ANALYZING THE INFLUENCE OF UNIVERSITY EDUCATION \\ ON THE ENTREPRENEURIAL PROFILE OF THE STUDENT
}

Guadalupe Oliveras², Hernán Pedro Vigier³ ${ }^{3}$ José Alberto Porras ${ }^{4}$

\section{Resumen}

El trabajo tiene el objetivo de evaluar el efecto de la educación universitaria (con y sin educación emprendedora en la currícula) en el perfil emprendedor de los estudiantes. Particularmente, se analiza cómo la percepción de factibilidad, la percepción de deseabilidad, el conocimiento emprendedor, el área de estudio de los estudiantes y el año de cursado, influyen en la intención emprendedora de los estudiantes.

Para ello se lleva a cabo un relevamiento en estudiantes universitarios de primero y último año de dos universidades públicas de la región. De las respuestas obtenidas se observa que incorporar educación emprendedora tiene una influencia positiva en la intención de emprender, generando un efecto contrario en los casos donde no se incluye. Además, los estudiantes que han recibido educación emprendedora (en comparación con los que no) reconocen haber afianzado sus conocimientos sobre el mundo emprendedor y haber desarrollado habilidades que podrían serles útiles en caso de decidirse a emprender. 
Palabras clave: educación emprendedora, intención emprendedora, universidad, emprendedorismo.

\begin{abstract}
The paper aims to evaluate the effects of university education (with and without entrepreneurial education) on the entrepreneurial profile of students. In particular, we analyze how entrepreneurial intention of students is influenced by the feasibility perception, desirability perception, entrepreneurial knowledge and the area of study.

For this purpose, we apply a survey in university students of two public universities. The results suggest that entrepreneurship education in the curricula has a positive influence on the entrepreneurial intention. In addition, students who have received entrepreneurship education (compared to those who did not) recognize have strengthened their knowledge of entrepreneurship and have developed entrepreneurial skills that could be useful if they decide to undertake a venture.
\end{abstract}

Keywords: entrepreneurial education, entrepreneurial intention, university, entrepreneurship.

JEL: L26

16 - Escritos Contables y de Administración 
Analizando la influencia de la educación universitaria en el perfil emprendedor...

\section{Introducción}

En la actualidad, el emprendedorismo es considerado como una herramienta fundamental para el motor de cualquier economía. Como respuesta a ello, algunas universidades y gobiernos han propiciado el desarrollo de valores y capacidades emprendedoras, sobre todo en la población universitaria. En este marco, comienzan a cobrar protagonismo investigaciones centradas en estudiar cuál es el impacto de los programas de educación emprendedora (Pittaway y Cope, 2007; Neck y Greene, 2011; Martín McNally y Kay, 2013; Walter, Parboteeah y Walter, 2013) y si efectivamente aquellas instituciones que no sumen ese desafío generan algún efecto contrario.

Dentro de la forma de evaluar la educación emprendedora cobraron protagonismo diferentes enfoques. En primera medida se consideraba correcto medir el número de emprendimientos en marcha y/o su impacto (McMullan y Long, 1987; Wilson, Vyakarnam, Volkmann, Mariotti y Rabuzzi, 2009). Sin embargo, según Fayolle y Degeorge (2006), la creación de empresas no puede ser medida durante o inmediatamente después de la formación específica, ya que el proceso de creación de empresas lleva su tiempo. A su vez, según los autores, cuanto más se retrasa la medición, más difícil es aislar el papel desempeñado por el efecto formativo de otras variables que han tenido influencia en el acto de creación. Por ello, surge la alternativa de medir los efectos a nivel interpersonal, analizando si los cursos y los programas sobre emprendedorismo promueven en los estudiantes las habilidades y las competencias necesarias para emprender con éxito, lo que incluye características personales y conocimiento específico relacionado con el mundo emprendedor (González López, García Piñeiro y Rodríguez Ariza, 2012). Así, se comienzan a aplicar los modelos de intención para analizar el impacto de este tipo particular de formación.

Los modelos dedicados a estudiar la intención emprendedora se desarrollaron como mecanismos destinados a predecir el comportamiento emprendedor. Si bien la intención se vuelve un elemento previo imprescindible (Liñán, 2004a), existen dudas respecto de si una medida de la intención será siempre una predicción de la conducta (Fishbein y Ajzen, 1980; Fishbein, 1990, en Reyes Rodríguez, 2007), pero sí indicará mayores 
probabilidades de que dicha conducta ocurra, pues la relación intenciónconducta no es ni lineal ni unidireccional (Carsrud y Brännback, 2011).

Frente a este escenario, el presente trabajo tiene como objetivo evaluar la intención emprendedora en estudiantes universitarios de la provincia de Buenos Aires, con y sin educación emprendedora incluida dentro de los planes de estudio. El trabajo cobra importancia pues no se han encontrado en la Argentina trabajos empíricos que evalúen los efectos de la educación universitaria en el perfil emprendedor de los estudiantes, comparando el perfil "tradicional" (de nivel universitario) con aquel que ha recibido educación emprendedora a través de un programa universitario donde el tema se aborda de manera transversal, modalidad que lo distingue de aquellos casos en los que se introduce una o algunas materias (obligatorias $\mathrm{u}$ optativas) con contenido emprendedor. Se pretende de este modo generar aportes concretos sobre lineamientos y consideraciones a tener en cuenta a la hora de decidir (o no) incorporar educación emprendedora en la currícula universitaria.

El trabajo se diseña como sigue: en una primera instancia, se presenta el marco teórico de referencia; luego, la metodología seguida, y por último la presentación de los resultados y las reflexiones finales.

\section{Marco teórico}

Los modelos más citados en lo que respecta a la intención emprendedora pertenecen a los aportes de Shapero (1982), quien desarrolló su teoría a través del Modelo del Evento Empresarial (EEM por sus siglas en inglés: Entrepreneurial Event Model), y los aportes de Ajzen (1987) mediante el Modelo de la Conducta Planificada (TPB por sus siglas en inglés Theory of Planned Behavior). Luego de ambos, se derivaron otros, como por ejemplo, Krueger y Brazeal (1994) y Liñán (2004b).

En los aportes de Shapero (1982), la intención de emprender es el resultado de dos percepciones: la percepción de deseabilidad y la percepción de factibilidad. Al respecto, afirma que percibir una opción en particular tanto deseable como factible genera una pequeña posibilidad de que la misma sea considerada. La percepción de deseabilidad es entendida como la 
probabilidad percibida por la persona de obtener resultados personalmente atractivos a través de la realización de la conducta de emprender. El resultado de esta percepción es producto de una matriz conformada por la cultura, la estructura socioeconómica, la familia y la educación, así como también por las personas influyentes de nuestro alrededor. La percepción de factibilidad, por su parte, es definida como el grado en que una persona considera personalmente posible llevar a cabo cierta conducta. De este modo, cualquier variable influirá sólo indirectamente a través de estas dos percepciones.

Años más tarde, Ajzen (1987) propone el Modelo de la Conducta Planificada. Según esta teoría, el comportamiento humano se guía por tres tipos de percepciones: las creencias acerca de los posibles resultados al realizar la conducta y sus evaluaciones (actitud hacia la conducta), las creencias acerca de las expectativas normativas de los demás y la motivación para cumplir con estas expectativas (percepción de las normas subjetivas), y las creencias acerca de la presencia de factores que puedan facilitar o impedir el desempeño de la conducta y el poder percibido de estos factores (percepción del control de una conducta). Según el autor, en combinación, la actitud hacia la conducta, la percepción de las norma subjetivas y la percepción de control de la conducta conducen a la formación de una intención de comportamiento, en este caso, hacia emprender. Como regla general, establece que cuanto más favorable sea la actitud hacia la conducta y la percepción de la norma subjetiva, y mayor sea la percepción de control de la conducta, más fuerte deberá ser la intención de la persona para realizar el comportamiento en cuestión.

A fin de unificar las variables propuestas por los dos autores, Krueger y Brazeal (1994) desarrollan el Modelo del Emprendedor Potencial. En este caso, consideran que los factores del ambiente, específicamente los factores informales como las actitudes hacia el emprendedorismo, afectan la decisión de crear una nueva empresa. Así, establecen que existen dos constructos críticos a la intención: la percepción de deseabilidad, que incluye la percepción de las normas sociales y la actitud hacia la conducta, y la percepción de factibilidad, relacionada con el concepto de control de la conducta.

Liñán (2004b), por su parte, establece que la intención de emprender depende, a su vez, de la deseabilidad y la factibilidad percibida hacia esa 
actividad y, de forma adicional, incluye el conocimiento emprendedor de la persona como un aspecto que ayuda a configurar la firmeza de esa intención o potencial. En esta línea, Liñán (2004b) propone introducir al modelo de Krueger y Brazeal (1994) el conocimiento sobre el marco institucional empresarial como una variable más, a la que denomina "conocimiento emprendedor". Según el autor, un mayor conocimiento del entorno emprendedor contribuirá sin duda a tener percepciones más realistas sobre el tema y proporcionará de manera directa una mayor conciencia de la existencia de esa opción profesional, lo que genera que la intención de convertirse en un emprendedor, se vuelva más creíble (Liñán, 2004b).

En este marco se proponen las siguientes hipótesis:

- Hipótesis 1: la percepción de deseabilidad de los estudiantes universitarios afecta positivamente a la intención de emprender.

- Hipótesis 2: la percepción de factibilidad de los estudiantes universitarios afecta positivamente a la intención de emprender.

- Hipótesis 3: el conocimiento emprendedor de los estudiantes universitarios afecta positivamente a la intención de emprender.

Por otro lado, a nivel general, Singh (1990; en Gorman, Hanlon y King, 1997) establece que la educación tradicional podría inhibir el emprendedorismo, lo que indicaría la necesidad de reorientar los sistemas educativos a fin de enfatizar y valorar la cuestión emprendedora, siempre que se pretenda cultivarla. De hecho, según Görisch (2002, en Ascúa, Andrés y Danko, 2014), particularmente el tipo de estudios que siguen los estudiantes universitarios influye en la intención de emprender.

Para el caso de la Argentina, Ussman y Postigo (2000) indican que la mayoría de las casas de altos estudios tienden generalmente a formar alumnos para que desarrollen una carrera como "empleados", y solo en raras ocasiones abordan la perspectiva de desarrollar competencias que permitan poner en marcha sus propios proyectos. De hecho, para 1996 solo el $4 \%$ del total de las universidades tenían programas en el área de emprendedorismo (Postigo y Tamborini, 2002). Después del año 2000, ese porcentaje asciende aproximadamente al 30 \% (Cesar, Fardelli Corropolese, Federico y Baruj, s.f.) y al mismo tiempo, a través de lo indicado en diferentes trabajos (Braidot, 2001; Postigo y Tamborini, 2002; Shiersmann, Graña, Liseras, 2002; Cutidiano, 
Cesar, Porras, Partal, 2006; Porras, Partal, Amadío, Murello, Cesar, 2006; Vigier, Partal, Savoretti, Porras, 2006; Porras, Oliveras, Vigier, Savoretti, Dichiara, Bruno, 2009), puede verse un compromiso cada vez mayor de las casas de altos estudios con el desarrollo de las competencias emprendedoras, asociado a un interés creciente por el fenómeno de la creación de empresas. De aquí surge preguntar si ese compromiso asumido es valorado por los estudiantes.

Por lo anterior, se establecen las siguientes hipótesis:

- Hipótesis 4: la educación universitaria que no incorpora formación emprendedora afecta negativamente a la intención emprendedora de los estudiantes.

- Hipótesis 5: la intención emprendedora cambia al ingresar y al egresar de la universidad

- Hipótesis 6: aquellos estudiantes que reciben formación emprendedora reconocen el esfuerzo por parte de la universidad en ese sentido.

\section{Metodología}

Para testear las hipótesis de investigación se emplea un enfoque cuantitativo, el cual, de acuerdo a Sampieri Hernández, Collado Fernández y Lucio Baptista (2010:4), es el que "usa recolección de datos para probar hipótesis con base en la medición numérica y el análisis estadístico para establecer patrones de comportamiento y probar teorías".

A continuación, se describe el universo de estudio, las variables y los modelos de análisis, el diseño de la recogida de datos, así como también las herramientas estadísticas utilizadas para analizarlos. 


\subsection{El universo de estudio}

Con el objetivo de evaluar la intención emprendedora en estudiantes con y sin educación emprendedora en su formación universitaria, se eligieron como universo de estudio a los estudiantes de primero y último año pertenecientes a dos de las universidades públicas con sede en el sudoeste de la provincia de Buenos Aires: la Universidad Nacional del Sur (UNS) y la Universidad Provincial del Sudoeste (UPSO).

Luego, se seleccionaron las áreas del conocimiento a considerar en el estudio. Para ello se siguieron clasificaciones de trabajos previos (Ascúa et al., 2014; Guerrero, Rialp y Urbano, 2008), incorporando una nueva área vinculada con la formación emprendedora para ubicar a aquellos estudiantes que sí habían recibido educación emprendedora dentro de su plan de estudios. Esta cuestión se diferencia de trabajos previos que consideraron a los estudiantes de ciencias económicas como aquellos pertenecientes al área emprendedora (Guerrero et al., 2008). De este modo, las áreas identificadas fueron: Ingeniería, Ciencias Naturales, Ciencias Económicas y Tecnicaturas Emprendedoras.

Dentro de Ingeniería (IN), se incluyeron a las carreras cuya formación se encuentra vinculada con aspectos técnicos y de las ciencias duras, tales como Ingeniería Civil, Ingeniería Industrial, Ingeniería Mecánica, Ingeniería Electricista, Ingeniería Electrónica, Ingeniería de Alimentos, Ingeniería Química, Ingeniería en Sistemas de la Computación, licenciatura en Ciencias de la Computación e Ingeniería Agronómica. La duración nominal promedio de estas carreras ronda los 5 años. Asimismo, se observa que dentro de su plan de carrera vigente al año 2012, no cuentan con ninguna formación explícitamente vinculada con la cuestión emprendedora, aunque algunas de las carreras incluyen asignaturas como evaluación de proyectos, evaluación económica de procesos, economía de la empresa y organización industrial.

Dentro de las Ciencias Naturales (CN), se ubicaron aquellas disciplinas científicas que se dedican al estudio de la naturaleza. De este modo, fue conformado por licenciatura en Ciencias Biológicas, Farmacia y Bioquímica. La duración nominal de estas carreras resulta ser de 5 años y no presentan, en 
los planes de carrera vigentes al año 2012, ninguna asignatura vinculada con economía, ni con la cuestión emprendedora.

En Ciencias Económicas (CE), por su parte, se incluyeron las carreras de grado relacionadas con el estudio de actividades económicas, tales como licenciatura en Administración, Contador Público y licenciatura en Economía, cuya duración promedio también ronda los 5 años. Respecto de la vinculación de sus contenidos con el área emprendedora, si bien tradicionalmente han sido carreras relacionadas con la temática, lo cierto es que de acuerdo a los planes de estudio vigentes al año 2012, los contenidos que tienen las asignaturas más vinculadas con la creación de empresas tienen que ver con la formulación y la evaluación de proyectos. No obstante, se destaca que este tipo de carreras presenta además otros contenidos que también resultan ser herramentales para la creación de empresas como costos, finanzas, comercialización, entre otras.

Por último, dentro de las Tecnicaturas Emprendedoras (TE), se incluyeron las carreras con un componente emprendedor en las currículas. De este modo se tuvieron en cuenta las tecnicaturas con perfil emprendedor pertenecientes a la UPSO y al Programa de Estudios Universitarios en la Zona (PEUZO) perteneciente a la UNS. En estas tecnicaturas, de tres años de duración, la cuestión emprendedora fluye trasversalmente $\mathrm{y}$, a su vez, presentan una temática de interés que las particulariza, y hace que todos los contenidos estén vinculados con ella. Allí se ha optado por un modelo aditivo de educación emprendedora, que busca capacitar a los estudiantes en un área temática de interés local, tanto en las cuestiones técnicas como en sus negocios asociados, fortalecer sus competencias emprendedoras necesarias para identificar y explotar las oportunidades y proporcionarles las herramientas y los conocimientos necesarios para crear una organización, y gestionar su funcionamiento y eventual crecimiento (Porras, Oliveras, Vigier, Savoretti, Dichiara, Bruno, 2009). Las carreras involucradas en el estudio fueron las tecnicaturas universitarias en Emprendimientos Turísticos, Asuntos Municipales, Emprendimientos Informáticos, Manejo y Comercialización de Granos, Gestión Cultural y Emprendimientos Culturales, Emprendimientos Agropecuarios, Creación y Gestión de PyMes, Emprendimientos Agroalimentarios y Emprendimientos Audiovisuales. 


\subsection{Recogida de datos y tamaño de la muestra}

Para determinar el tamaño de la muestra, se definió como estudiantes de "primer año" a aquellos alumnos que habían cursado al menos una materia del primer cuatrimestre del primer año de su plan de estudios, mientras que como alumnos de "último año", a aquellos que habían cursado al menos una materia en el primer cuatrimestre del último año de su plan de estudios. Luego, se solicitó información tanto a la Secretaría Académica de la UNS como de la UPSO. Así, para julio de 2011 la población total de estudiantes de primer año del segundo cuatrimestre, pertenecientes a las áreas de estudio bajo análisis, era de 1117 estudiantes, de los cuales 450 pertenecían al área de I, 265 al área de CN, 321 al área de CE y 141 al área de TE. Por su parte, la cantidad de estudiantes objeto de estudio del último año alcanzaba un total de 1379 estudiantes, conformados por 475 del área de I, 200 del área de $\mathrm{CN}, 476$ del área de CE y 87 del área de TE. Teniendo en cuenta un error muestral del $5 \%$, un nivel de confianza del $95 \%$, la muestra resultó de 613 estudiantes universitarios en total.

El relevamiento fue llevado a cabo entre los meses de octubre, noviembre y diciembre del año 2011. Los datos de la encuesta fueron recabados aleatoriamente por dos vías: correo electrónico y contacto personal. Respecto del contacto personal, se tuvieron en cuenta todos los horarios de clase de las materias de primero y del último año pertenecientes al segundo cuatrimestre de acuerdo al plan de estudios.

\subsection{Definición de las variables de interés y del modelo de análisis}

Las variables fueron definidas siguiendo los modelos de intención emprendedora. Para ello se utilizaron variables cualitativas ordinales y nominales (categóricas y dicotómicas). Con las variables ordinales se buscó medir actitudes. Las actitudes son definidas como la predisposición de una persona para responder coherentemente de manera favorable o desfavorable ante un objeto, ser vivo, actividad, concepto, persona o sus símbolos (Sampieri Hernández et al., 2010). Las actitudes solo son un indicador de la conducta, pero no de la conducta en sí (ibíd.), por lo si se detecta que la predisposición 
de los estudiantes al emprendedorismo es favorable, esto no significa que estén tomando acciones para emprender, aunque sí constituye un indicador de que pueden adoptarlas en algún momento. Pueden ser "semillas" que bajo ciertas condiciones pueden "germinar en comportamiento" (ibíd.).

Para mediar la intención emprendedora, siguiendo a Liñán (2008), se empleó una escala Likert de 7 puntos, donde 7 indicaba la máxima preferencia y 1 indicaba la mínima preferencia a tener un emprendimiento luego de graduarse. Sin embargo, para efectuar las correspondientes regresiones, la codificación fue modificada en tres posibles resultados: alto (se ubicaron aquellas respuestas con un puntaje de 6 o 7); medio (se ubicaron aquellas respuestas con un puntaje de 3,4 o 5) y bajo (se ubicaron aquellas respuestas con un puntaje de 1 o 2).

Respecto de las variables explicativas, contemplando los modelos descritos en el marco teórico, se consideraron como variables independientes: la percepción de deseabilidad, la percepción de factibilidad, la percepción del conocimiento emprendedor, el área de estudio y la condición de alumno (ingresante o por egresar).

La percepción de deseabilidad (des), siguiendo a Guerrero et al. (2008), es entendida como cuán atractiva encuentra el estudiante la idea de iniciar su propio negocio. Se definió como variable cualitativa ordinal, empleando en este caso una escala Likert de 7 puntos, donde 7 indica la máxima atractividad y 1 la mínima. La percepción de factibilidad (fac), siguiendo nuevamente el trabajo de Guerrero et al. (2008), es entendida como el grado de confianza autopercibida sobre las propias habilidades y capacidades para iniciar un negocio. Es definida como variable cualitativa ordinal, empleando en este caso una escala Likert de 7 puntos, donde 7 indica la máxima confianza y 1 la mínima.

Para la percepción del conocimiento emprendedor (con), se emplearon tres indicadores referidos al grado de conocimiento sobre políticas de estado para financiar emprendimientos, incubadoras de empresas y programas existentes de apoyo a emprendedores. Estos indicadores fueron definidos como variable cualitativa ordinal, empleando en este caso una escala Likert de 7 puntos, donde 7 indica el máximo conocimiento y 1 indica el mínimo. En este caso, dado que se trata de una variable medida a través de varios indicadores, es 
recomendable que se analice la confiabilidad de la misma. Al respecto, Hernández Sampieri et al. (2010) señalan que uno de los métodos más aplicados es el Alfa de Cronbach, aclarando que si bien no hay una regla que indique la interpretación del mismo, se puede decir de manera más o menos general, que un resultado de 0.25 indica baja confiabilidad; si es 0.50 , la fiabilidad es media o regular; si supera el 0.75, es aceptable; y si es mayor a 0.90 , se considera elevada. En este caso, se obtuvo 0.881 , por lo que, siguiendo el criterio anterior, se considera aceptable.

Respecto de las áreas del conocimiento, teniendo en cuenta la clasificación realizada con anterioridad (IN, CN, CE y TE), se las denominó Área 1, Área 2, Área 3 y Área 4 respectivamente. A los fines del modelo, se utilizan tres variables dummy (Área 1, Área 2, Área 3) que pueden asumir dos valores de acuerdo al grado de pertenencia: $0=$ no pertenece y $1=$ pertenece. De este modo, cuando Área 1 asume valores iguales a uno (Área $1=1$ ), se compara estudiantes del área de IN con el resto de los perfiles; cuando Área 2 asume valores iguales a uno (Área $2=1$ ), se compara estudiantes del área de $\mathrm{CN}$ con el resto de los perfiles; cuando Área 3 asume valores iguales a uno (Área $3=$ 1 ), se compara estudiantes de CE con el resto de los perfiles. Cuando las tres variables asumen valores iguales a cero, se analizan los estudiantes pertenecientes al área TE (representando de este modo los datos pertenecientes al Área 4).

Por último, respecto de la condición de alumno (condi) corresponde a la transición del estudiante a lo largo de la carrera universitaria. Bajo el supuesto de continuidad en el ciclo formativo se relevan dos casos: al inicio y al final de la carrera. Así, esta variable se definió como dicotómica, que asume valores iguales a cero cuando el encuestado es ingresante y valores iguales a uno cuando se trata de un alumno del último año de la carrera.

Por su parte, como los factores listados anteriormente no son los únicos que se espera influyan en la intención emprendedora, se incorporan también una serie de variables de control que cumplen el rol de neutralizar los efectos sobre la variable dependiente: el género y la edad. Respecto del género, la variable tomó valores 0 en caso de pertenecer al género masculino, y 1 en caso de pertenecer al género femenino. Por su parte, la edad fue definida como variable cuantitativa y se refiere a la edad del encuestado. 
De este modo, se definió como modelo a testear el que sigue:

$$
Y(I E)=f(\text { sexo, edad, condi, área } 1 \text {, área } 2 \text {, área3, des, fac, con })
$$

A fin de llevar a cabo el procesamiento de los datos, se utilizaron modelos econométricos de variable dependiente cualitativa, a saber: Multinomial Logit, Ordered Logit y Generalized Ordered Logit. En este caso, se aplicó como software el programa Stata 7.0.

Asimismo, para analizar la vinculación entre las áreas de estudio y los contenidos vinculados en el caso de que se decidiera emprender, se utilizó la tabla de contingencia y su correspondiente análisis de significatividad. Para el siguiente trabajo, el nivel de significatividad se define del 10\%, del 5\% y del $1 \%$ y se señala del siguiente modo $10 \%$ con *, al $5 \%$ con **, y al 1\% con ***. Se aclara además que en el análisis descriptivo el p-valor solo es presentado en el caso de resultar significativo.

\section{Resultados}

De la propia sistematización de los datos, fueron descartados de la muestra 8 cuestionarios por no contar con todas las respuestas completas. De este modo, el total de cuestionarios analizados fueron 592, 300 de primer año y 292 del último año. A continuación, se presenta un análisis descriptivo para luego dar lugar a los resultados producto de las estimaciones.

\subsection{Análisis descriptivo}

Los datos descriptivos de la muestra se presentan en la tabla 1. Allí se observa que la población estudiantil bajo análisis está conformada en mayor medida por estudiantes de género femenino, pues aproximadamente el $58 \%$ cumple con esta condición. Sin discriminar por año de cursado, ocurre lo mismo para las áreas de CN (72,07\%), CE (72,97\% ) y TE (67,29\%). No así en el área de IN en donde, como tradicionalmente ha sucedido, priman estudiantes de género masculino. 
Guadalupe Oliveras, Hernán Pedro Vigier, José Alberto Porras

Tabla 1. Características generales de la muestra

\begin{tabular}{lccccc}
\multicolumn{1}{c}{ Variables } & Total & IN & CN & CE & TE \\
\cline { 2 - 6 } Tamaño de la muestra & 592 & 263 & 111 & 111 & 107 \\
-Primer año & 300 & 117 & 53 & 74 & 56 \\
-Último año & 292 & 146 & 58 & 37 & 51 \\
\hline Género Femenino & $58,28 \%$ & $42,59 \%$ & $72,07 \%$ & $72,97 \%$ & $67,29 \%$ \\
-Primer año & $62,67 \%$ & $52,14 \%$ & $66,04 \%$ & $77,03 \%$ & $62,50 \%$ \\
-Último año & $53,77 \%$ & $34,93 \%$ & $77,59 \%$ & $64,86 \%$ & $72,55 \%$ \\
\hline Edad promedio & 22,20 & 21,70 & 21,86 & 20,32 & 25,76 \\
-Primer año & 20,15 & 18,99 & 18,87 & 19,30 & 24,91 \\
-Último año & 24,31 & 23,86 & 24,60 & 22,35 & 26,69 \\
\hline Padre emprendedor & $38,51 \%$ & $40,68 \%$ & $35,14 \%$ & $42,34 \%$ & $32,71 \%$ \\
-Primer año & $36,67 \%$ & $37,61 \%$ & $33,96 \%$ & $39,19 \%$ & $33,93 \%$ \\
-Último año & $40,41 \%$ & $43,15 \%$ & $36,21 \%$ & $48,65 \%$ & $31,37 \%$ \\
\hline Consideran que son muy & $49,10 \%$ & $50,96 \%$ & $51,28 \%$ & $52,17 \%$ & $36,36 \%$ \\
buenos emprendedores & $58,72 \%$ & $65,12 \%$ & $61,11 \%$ & $60,00 \%$ & $38,89 \%$ \\
-Primer año & $39,82 \%$ & $40,98 \%$ & $42,86 \%$ & $37,50 \%$ & $33,33 \%$ \\
\hline -Último año & $19,76 \%$ & $20,91 \%$ & $20,72 \%$ & $13,51 \%$ & $22,43 \%$ \\
\hline Madre emprendedora & $17,67 \%$ & $18,80 \%$ & $15,09 \%$ & $16,22 \%$ & $19,64 \%$ \\
-Primer año & $21,92 \%$ & $22,60 \%$ & $25,86 \%$ & $8,11 \%$ & $25,49 \%$ \\
-Último año & $34,21 \%$ & $31,48 \%$ & $40,91 \%$ & $50,00 \%$ & $25,00 \%$ \\
\hline Consideran que son muy & $43,14 \%$ & $28,57 \%$ & $37,50 \%$ & $63,64 \%$ & $54,55 \%$ \\
buenas emprendedoras & $26,98 \%$ & $33,33 \%$ & $42,86 \%$ & $0,00 \%$ & $0,00 \%$ \\
-Primer año & & & & & \\
-Último año & & & & \\
\hline
\end{tabular}

Fuente: elaboración propia. 
En lo que hace a la edad, si bien resultaría esperable que para todas las áreas sean similares las edades en los estudiantes de primer y último año de las carreras, es notable en este caso que los estudiantes pertenecientes al área TE presentan mayor edad en comparación con las otras áreas 5 . Mientras que en ese grupo la edad promedio para primer año ronda los 25 años, el resto oscila en los 19 años.

En cuanto a la existencia de un microentorno emprendedor, esto es, la presencia de padre o madre que haya llevado a cabo su propio emprendimiento, se encuentra que, a nivel general, más de la mitad no cuenta con estos modelos de rol. En cuanto a la parte paterna, los estudiantes que manifiestan mayor presencia son los pertenecientes al área de CE $(42,34 \%$ del total, $39,19 \%$ los de primer año y $48,65 \%$ los del último año), mientras que la menor cantidad se encuentra en los estudiantes de TE $(32,71 \%$ del total, $33,93 \%$ los de primer año y $31,37 \%$ los del último año). Por su parte, también resulta baja la presencia de madres emprendedoras. Para el total de la muestra, solo aproximadamente el 19\% cumple con esta condición.

Por último, en lo que hace a la calificación dada por los estudiantes a los padres y/o madres que crearon su empresa, se encuentra que mientras el $49,10 \%$ está de acuerdo con la calificación de "buen emprendedor" en referencia a su padre, un porcentaje mucho menor $(34,21 \%)$ lo está en referencia a su madre. La misma diferencia se puede observar para todas las áreas de estudio consideradas en el trabajo (50,96 \% y 31,48\% \% para el caso de IN; $51,28 \%$ y $40,91 \%$ en CN; $52,17 \%$ y $50,00 \%$ para CE; $36,36 \%$ y $25 \%$ para las TE). Esta última cuestión indicaría que a nivel general la valoración social dada a los emprendedores de género masculino continúa siendo superior a la valoración de las mujeres que deciden emprender.

\subsection{Análisis de regresión}

En la tabla 2 se presentan los valores medios de las variables de interés, según el grado de intención. Los resultados muestran que los estudiantes más jóvenes son los que se ubican en el grado medio o indeciso de intención emprendedora, mientras que los más grandes parecieran tener una decisión más terminada, que puede coincidir con sus preferencias profesionales para 
luego de graduarse. Por su parte, se observa una mayor participación de las mujeres en los tres grupos aunque su participación se ve disminuida cuando se trata del grado más alto de intención emprendedora. Asimismo, tal como se esperaba, el grupo con mayor grado de intención emprendedora presenta un alto porcentaje de alta percepción de deseabilidad y alta percepción de factibilidad, no así de conocimiento emprendedor.

Por último, es posible observar que el grado más alto de intención emprendedora está conformado en mayor medida por estudiantes del área de IN, seguido por los de las TE, CE y en menor proporción por estudiantes del área de $\mathrm{CN}$.

Tabla 2. Estadística descriptiva según grado de intención

\begin{tabular}{lccc}
\cline { 2 - 4 } & \multicolumn{1}{c}{$\gamma=1$} & $\gamma=2$ & $\gamma=3$ \\
\hline Edad & 22,16 años & 21,79 años & 22,38 años \\
Primer año de cursado & $42,47 \%$ & $62,50 \%$ & $53,07 \%$ \\
Último año de cursado & $57,53 \%$ & $37,50 \%$ & $46,93 \%$ \\
Género femenino & $59,36 \%$ & $59,38 \%$ & $57,04 \%$ \\
Género masculino & $40,64 \%$ & $40,63 \%$ & $42,96 \%$ \\
Área 1 & $56,16 \%$ & $45,83 \%$ & $34,66 \%$ \\
Área 2 & $15,07 \%$ & $23,96 \%$ & $19,86 \%$ \\
Área 3 & $15,53 \%$ & $17,71 \%$ & $21,66 \%$ \\
Área 4 & $13,24 \%$ & $12,50 \%$ & $23,83 \%$ \\
Alta percepción de factibilidad & $50,23 \%$ & $41,67 \%$ & $74,01 \%$ \\
Alta percepción de deseabilidad & $67,12 \%$ & $75 \%$ & $90,61 \%$ \\
Alto conocimiento emprendedor & $14,61 \%$ & $13,54 \%$ & $29,60 \%$ \\
\hline
\end{tabular}

Fuente: elaboración propia.

En cuanto a los coeficientes productos de las estimaciones del modelo, los resultados se pueden ver en la tabla 3. Cabe aclara que, para captar el efecto diferencial entre las tres categorías, se plantea el modelo usando primero a $Y$ $=1$ como categoría base y luego $\mathrm{Y}=2$. 
Analizando la influencia de la educación universitaria en el perfil emprendedor...

Tabla 3. Estimaciones Modelo (MNLM, OLM, GOLM)

\begin{tabular}{|c|c|c|c|c|c|c|}
\hline \multirow{4}{*}{ Variables explicativas } & \multirow{2}{*}{\multicolumn{3}{|c|}{ MNLM }} & \multirow{4}{*}{$\begin{array}{c}\text { OLM } \\
\text { Sin base }\end{array}$} & \multirow{3}{*}{\multicolumn{2}{|c|}{$\begin{array}{c}\text { GOLM } \\
\text { Base: Grupo } 3\end{array}$}} \\
\hline & & & & & & \\
\hline & \multicolumn{2}{|c|}{ Base: Grupo 1} & \multirow{2}{*}{$\begin{array}{c}\text { Base: Grupo } 2 \\
\text { Grupo 3-2 }\end{array}$} & & & \\
\hline & Grupo 2-1 & Grupo 3-1 & & & Grupo 1-2 & Grupo 2-3 \\
\hline $\begin{array}{l}\text { Percepción de } \\
\text { deseabilidad }\end{array}$ & 0,1360 & 0,3340 & 0.1981 & 0,2180 & 0,2072 & 0,2230 \\
\hline $\mathrm{p}$ - value & 0,1690 & $0,0110^{* *}$ & $0.0640^{*}$ & $0,0050^{* * *}$ & $0,0340^{* *}$ & $0,0290^{* *}$ \\
\hline $\begin{array}{l}\text { Percepción de } \\
\text { factibilidad }\end{array}$ & 0,2281 & 0,4385 & 0.2104 & 0,2794 & 0,2872 & 0,2602 \\
\hline $\mathrm{p}$-value & $0,014^{* *}$ & $0,0000^{* * *}$ & $0.0220^{* *}$ & $0,0000^{* * *}$ & $0,0020^{* * *}$ & $0,0030^{* * *}$ \\
\hline $\begin{array}{l}\text { Conocimiento } \\
\text { emprendedor }\end{array}$ & 0,2900 & 0,5275 & 0.2374 & 0,3169 & 0,3575 & 0,2895 \\
\hline $\mathrm{p}$-value & $0,001^{* * *}$ & $0,0000^{* * *}$ & $0.0010^{* *}$ & $0,0000^{* * *}$ & $0,0000^{* * *}$ & $0,0000^{* * *}$ \\
\hline Área 1 & 0,4908 & $-0,2978$ & -0.7885 & $-0,4095$ & 0,1655 & $-0,6902$ \\
\hline $\mathrm{p}$-value & 0,2690 & 0,5500 & $0.0220^{* *}$ & 0,1590 & 0,6980 & $0,0380^{* *}$ \\
\hline Área 2 & 0,0446 & $-1,1300$ & -1.1746 & $-0,8887$ & $-0,3897$ & $-1,1410$ \\
\hline $\mathrm{p}$-value & 0,9090 & $0,0100^{* * *}$ & $0.0000^{* * *}$ & $0,0010^{* * *}$ & 0,2950 & $0,0000^{* * *}$ \\
\hline Área 3 & 0,4033 & $-0,4028$ & -0.8060 & $-0,5019$ & 0,0392 & $-0,7332$ \\
\hline $\mathrm{p}$-value & $-0,3900$ & 0,4310 & $0.0170^{* *}$ & $0,0900^{* *}$ & 0,9310 & $0,0250^{* *}$ \\
\hline Edad & $-0,0186$ & $-0,0104$ & 0.0083 & $-0,0011$ & $-0,1676$ & 0,0049 \\
\hline $\mathrm{p}$-value & 0,605 & 0,7830 & 0.7380 & 0,9610 & 0,6090 & 0,8350 \\
\hline Sexo & $-0,3259$ & $-0,4954$ & -0.1695 & $-0,2604$ & $-0,3333$ & $-0,2278$ \\
\hline $\mathrm{p}$-value & 0,22 & 0,1110 & 0.4400 & 0,1430 & 0,2000 & 0,2880 \\
\hline Condición & 0,4908 & 0,1964 & -0.2943 & 0,0394 & 0,3818 & $-0,2028$ \\
\hline $\mathrm{p}$ - value & $0,0850^{*}$ & 0,5480 & 0.2070 & 0,8380 & 0,1700 & 0,3760 \\
\hline
\end{tabular}

Fuente: elaboración propia.

Cuando se emplea el método de estimación de MNLM es posible apreciar que el grupo con alta intención emprendedora se diferencia claramente de los estudiantes con baja intención en lo que hace a la percepción de deseabilidad, de factibilidad y al conocimiento emprendedor. De todas formas, tal como se conoce, el MNLM establece que las salidas no tienen un orden natural y, dado que no es lo que sucede en este caso en particular, que se aplican los otros dos métodos (OLM y GOLM). Allí se observa que la percepción de deseabilidad, de factibilidad y el conocimiento emprendedor resultan positivas y significativas. En ambos casos, se observa además que las áreas del conocimiento resultan negativas y significativas para pasar de una intención media a una intención emprendedora alta, lo que coincide con lo obtenido con el MNLM cuando se emplea como base $Y=2$. 
Para la interpretación de los coeficientes, es necesario el cálculo de los odds ratios, pues permiten dar una interpretación intuitiva de los mismos. Los odds ratios representan el cociente de probabilidades entre que ocurra un suceso respecto de que no ocurra. En este caso, a fin de no repetir las estimaciones, dichas probabilidades se calculan con el modelo de regresión más completo, es decir el GOLM.

En la tabla 4 se presenta el análisis de los odds ratios, considerando solo aquellos que resultaron significativos.

Tabla 4. Estimación de los odds ratios

\begin{tabular}{|c|c|c|}
\hline \multirow{4}{*}{$\begin{array}{c}\text { Modelo } \\
\text { Variables explicativas }\end{array}$} & \multirow{2}{*}{\multicolumn{2}{|c|}{ GOLM }} \\
\hline & & \\
\hline & \multicolumn{2}{|c|}{ Base: Grupo 3} \\
\hline & Grupo 1-2 & Grupo 2-3 \\
\hline Percepción de deseabilidad & 1.2302 & 1.2497 \\
\hline$p$-value & $0.034^{* *}$ & $0.029^{* *}$ \\
\hline Percepción de factibilidad & 1.3327 & 1.2972 \\
\hline $\mathrm{p}$-value & $0.002^{* * *}$ & $0.003^{* * *}$ \\
\hline Conocimiento emprendedor & 1.4297 & 1.3357 \\
\hline $\mathrm{p}$ - value & $0.000^{* * *}$ & $0.000^{* * *}$ \\
\hline Área 1 & 1.180 & 0.5014 \\
\hline $\mathrm{p}$ - value & 0.698 & $0.038^{* *}$ \\
\hline Área 2 & 0.6772 & 0.3194 \\
\hline $\mathrm{p}$ - value & 0.295 & $0.000^{* * *}$ \\
\hline Área 3 & 0.9615 & 0.4803 \\
\hline p-value & 0.931 & $0.025^{* *}$ \\
\hline Edad & 0.983 & 1.004 \\
\hline $\mathrm{p}$-value & 0.609 & 0.835 \\
\hline Sexo & 0.7165 & 0.7963 \\
\hline $\mathrm{p}$ - value & 0.200 & 0.288 \\
\hline Condición & 1.4648 & 0.8164 \\
\hline $\mathrm{p}$ - value & 0.170 & 0.376 \\
\hline
\end{tabular}

Fuente: elaboración propia. 


\subsubsection{Percepción de deseabilidad}

De acuerdo con los resultados obtenidos, se puede decir que si un estudiante incrementa su percepción de deseabilidad por tener un emprendimiento, se esperaría que las chances de pertenecer a $Y=2$ en comparación a $Y=1$ se incrementaran por un factor de 1.23. Asimismo, se puede observar que el impacto es similar si se cambia de una media a una alta intención. En este caso, si un sujeto incrementa su percepción de deseabilidad, se esperaría que las probabilidades de pertenecer a $Y=3$ en comparación a $Y=2$ se incrementaran por un factor de 1.24.

\subsubsection{Percepción de factibilidad}

En este caso se obtiene que si un sujeto incrementa su percepción de factibilidad por tener un emprendimiento, se esperaría que las probabilidades de pertenecer a $Y=2$ en comparación con $Y=1$ se incrementara por un factor de 1.3327. Asimismo, se puede ver que el impacto es apenas menor si se cambia de una media a una alta intención. En este caso, si un sujeto incrementa su percepción de factibilidad, se esperaría que las chances de pertenecer a $Y=3$ en comparación a $Y=2$ se incrementa por un factor de 1.297.

\subsubsection{Conocimiento emprendedor}

Respecto de esta variable, se puede decir que si un estudiante incrementa su conocimiento en el tema emprendedor, se esperaría que las probabilidades de pertenecer a $Y=2$ en comparación a $Y=1$ se incrementara por un factor de 1.429. Además, se puede observar que el impacto es escasamente menor si se cambia de una media a una alta intención. En este caso, si un estudiante incrementa su conocimiento en el tema emprendedor, se esperaría que las chances de pertenecer a $Y=3$ en comparación a $Y=2$ se incrementara por un factor de 1.3357. 


\subsection{4. Áreas del conocimiento}

Si se analizan ahora las áreas del conocimiento, llama la atención -en contraste con otros estudios realizados en la Argentina ${ }^{6}$ (Ascúa et al., 2014) que dichas áreas reducirían las chances para incrementar la intención emprendedora de los estudiantes, de media a alta.

Particularmente, se observa que pertenecer tanto al Área 1, al Área 2 como al Área 3, reduciría las chances de pasar de una media a una alta intención emprendedora, es decir, de $Y=2$ a $Y=3$, por un factor de 0.5 , 0.31 y 0.48 respectivamente.

\subsection{Análisis de la percepción de los estudiantes sobre el tipo de formación}

En lo que hace a la percepción de los estudiantes sobre la formación recibida, solo se tuvieron en cuenta las respuestas de los del último año. Se considera en este punto que los ingresantes no han transitado el total del camino como para tener una opinión más acabada.

En la tabla 5 se exponen los diferentes resultados, extrayendo para cada variable el porcentaje de estudiantes con alto grado de acuerdo sobre los contendidos otorgados durante su formación. Allí es posible observar rápidamente la diferencia entre los perfiles analizados. 
Analizando la influencia de la educación universitaria en el perfil emprendedor...

Tabla 5. Percepción sobre la formación recibida en lo que hace a la creación de empresas

\begin{tabular}{|c|c|c|c|c|c|}
\hline & \multicolumn{5}{|c|}{ Áreas } \\
\hline & Total & $I N$ & $C N$ & $C E$ & $T E$ \\
\hline $\begin{array}{l}\text { He adquirido herramientas } \\
\text { útiles si en algún momento de } \\
\text { mi vida deseo crear mi propia } \\
\text { empresa } \\
(\mathrm{p} \text {-value:0.000) })^{* * *}\end{array}$ & $37.24 \%$ & $31.03 \%$ & $24.14 \%$ & $45.95 \%$ & $64.00 \%$ \\
\hline $\begin{array}{l}\text { Siento que me ha preparado } \\
\text { más para trabajar en una gran } \\
\text { empresa que para crear mi } \\
\text { emprendimiento }\end{array}$ & $37.93 \%$ & $40.00 \%$ & $39.66 \%$ & $40.54 \%$ & $28.00 \%$ \\
\hline $\begin{array}{l}\text { Me ha dejado en claro que } \\
\text { tener mi propio } \\
\text { emprendimiento puede ser } \\
\text { una alternativa profesional } \\
\text { posible } \\
(\mathrm{p} \text {-value:0.000) } * * *\end{array}$ & $46.21 \%$ & $39.31 \%$ & $39.66 \%$ & $43.24 \%$ & $76.00 \%$ \\
\hline $\begin{array}{l}\text { He afianzado mi } \\
\text { conocimiento sobre el mundo } \\
\text { emprendedor } \\
\text { (p-value:0.000) }{ }^{* *}\end{array}$ & $23.79 \%$ & $12.41 \%$ & $22.41 \%$ & $13.51 \%$ & $66 \%$ \\
\hline $\begin{array}{l}\text { Pude extraer algunas } \\
\text { recomendaciones sobre las } \\
\text { buenas prácticas a la hora de } \\
\text { emprender. } \\
\text { (p-value:0.000) }{ }^{* * *}\end{array}$ & $39.31 \%$ & $31.72 \%$ & $41.38 \%$ & $18.92 \%$ & $74 \%$ \\
\hline $\begin{array}{l}\text { Me ha permitido desarrollar } \\
\text { habilidades necesarias para } \\
\text { ser un emprendedor } \\
\left(\mathrm{p} \text {-value:0.000) }{ }^{* * *}\right.\end{array}$ & $25.86 \%$ & $17.93 \%$ & $18.97 \%$ & $21.62 \%$ & $60 \%$ \\
\hline $\begin{array}{l}\text { Algún docente me transfirió } \\
\text { conocimientos y experiencias } \\
\text { en el tema emprendedor } \\
(p-v a l u e: 0.000)^{* * *}\end{array}$ & $37.93 \%$ & $33.10 \%$ & $29.31 \%$ & $21.62 \%$ & $74.00 \%$ \\
\hline
\end{tabular}

Fuente: elaboración propia.

Particularmente, en lo que hace a la adquisición de herramientas útiles si en algún momento desean crear su propia empresa, se observa para el total de la muestra de alumnos del último año que menos del $40 \%$ está de acuerdo con esta afirmación. Por su parte, mientras que los de Ciencias Naturales son los que menor porcentaje representan $(24.14 \%)$, los estudiantes de las TE son 
los que mayor participación tienen, de hecho, más del $60 \%$ está de acuerdo con esta afirmación (64\%). Asimismo, para esta variable se encontraron diferencias significativas, lo que pone en evidencia que para la muestra seleccionada la percepción sobre la adquisición de herramientas está vinculada con la formación recibida.

Respecto de la consideración sobre si la formación los ha preparado para trabajar en una gran empresa más que para crear su emprendimiento, se encuentra que casi el $40 \%$ de los estudiantes de IN, CN y CE están de acuerdo con esta afirmación. Por su parte el $28 \%$ de los estudiantes de las TE comparten esa posición. De todas formas, no se encuentran diferencias significativas para esas variables.

En relación con la percepción vinculada con el hecho de haber dejado en claro que tener su propio emprendimiento puede ser una alternativa profesional posible, se obtienen diferencias significativas, con lo cual tendría vinculación con el área de estudios. Se destaca que menos de la mitad del total de la muestra (el $46.21 \%$ ) está totalmente de acuerdo con esta afirmación. Por su parte, el porcentaje que sobresale en este punto es el de las TE (76\%), lo que de algún modo se ve traducido en la variable destinada a evaluar las preferencias profesionales. Aquí era posible observar que los estudiantes pertenecientes a esta área presentaban un perfil más abierto a otras opciones profesionales.

En cuanto al hecho de haber afianzado su conocimiento emprendedor, se encuentran diferencias significativas entre las distintas áreas y esta variable. Mientras que para el total de la muestra el $23.79 \%$ está totalmente de acuerdo con esta afirmación, el porcentaje en los estudiantes de las TE resulta ser casi tres veces superior (66\%). De este modo, parecería que los estudiantes confirmasen la formación recibida para tal fin.

En lo referido a las recomendaciones extraídas durante su formación sobre las buenas prácticas a la hora de emprender, son destacables dos puntos: por un lado, el bajo porcentaje otorgado por los estudiantes de CE a esta variable; por otro, el alto porcentaje encontrado en los estudiantes del área de las TE.

Por último, en cuanto al desarrollo de habilidades necesarias para ser un emprendedor y a la transferencia de conocimientos y experiencias en el tema durante el proceso de formación, es posible observar para ambas variables 
bajos porcentajes de acuerdo en las carreras que no han tenido formación emprendedora. Por su parte, se destacan mayores porcentajes de acuerdo en TE.

Para estas últimas cuatro variables analizadas se han encontrado diferencias significativas, lo que permite afirmar, para la muestra seleccionada, que la formación emprendedora es posteriormente reconocida por los estudiantes.

De todos los resultados encontrados, se halla un dato muy interesante, que corrobora algunas afirmaciones de otros autores (Singh, 1990 en Gorman et al., 1997; Ussman y Postigo, 2000; Postigo y Tamborini, 2002). Por un lado, casi más de la mitad de los estudiantes en general comienza con una preferencia por tener su propio emprendimiento en primer año (tabla 5)7, y por otro lado, esta cuestión pareciera no ser capitalizada por el tipo de educación recibida.

\subsection{Análisis de los resultados y las hipótesis de investigación}

Con el objetivo de reflexionar sobre los resultados encontrados, a continuación se presentan las hipótesis de investigación y se muestran las respuestas obtenidas, a fin de aceptar o rechazar las correspondientes hipótesis.

- Hipótesis 1: la percepción de deseabilidad de los estudiantes universitarios afecta positivamente a la intención de emprender.

- Hipótesis 2: la percepción de factibilidad de los estudiantes universitarios afecta positivamente a la intención de emprender.

- Hipótesis 3: el conocimiento emprendedor de los estudiantes universitarios afecta positivamente a la intención de emprender.

Respecto de la percepción de factibilidad y de deseabilidad, se encuentra fuerte apoyo a su favor de una respuesta positiva a esta pregunta. En todas las estimaciones se obtiene para cada una de estas variables, el signo esperado (positivo) y p-value significativo. Esto coincide fuertemente con los estudios previos que afirman que a medida que aumenta la percepción de factibilidad y deseabilidad, aumenta la intención a emprender. De todas formas es posible notar entre los coeficientes que la posibilidad de tener su propio emprendimiento les resulta a los estudiantes más factible que deseable, lo que 
no coincide con otros estudios que generalmente ponderan lo atractivo de esta opción laboral por encima de lo posible o realizable (Krueger et al., 2000; Guerrero et al., 2008; Liñán et al., 2011; Vigier, Jiménez, Oliveras, 2011).

En lo referido al conocimiento emprendedor, la evidencia encontrada en el presente estudio apoya fuertemente esta hipótesis. Los coeficientes encontrados para esta variable resultan fuertemente significativos para todas las opciones de modelos utilizados. Esta cuestión daría evidencias de que brindar conocimiento sobre la temática emprendedora aumenta la probabilidad de que los estudiantes incrementen su intención emprendedora.

A partir de lo anterior, las hipótesis 1, 2 y 3 resultan aceptadas.

- Hipótesis 4: la educación universitaria que no incorpora formación emprendedora afecta negativamente a la intención emprendedora de los estudiantes.

Las evidencias para la muestra seleccionada manifiestan, para los tres métodos de estimación, que la educación que no incorpora educación emprendedora a sus currículas afecta la intención de emprender, sobre todo para pasar de una intención media a una intención alta. Todos los coeficientes obtenidos de las áreas sin educación emprendedora resultan significativos y con signo negativo. Asimismo, tal como se pudo observar en la tabla 5, pertenecer al Área 1, Área 2 y Área 3 reduciría las chances de pasar de $Y=2$ a $\mathrm{Y}=3$. De este modo, la hipótesis 4 resulta también aceptada.

- Hipótesis 5: la intención emprendedora cambia al ingresar y al egresar de la universidad.

Para evaluar esta cuestión, se consideró para el análisis la comparación entre la intención emprendedora de los estudiantes de primero y del último año. Con las limitaciones del caso, no se está en condiciones de aceptar esta hipótesis. La variable elegida no resulta significativa para ninguno de los análisis, salvo para pasar de una baja intención a una media, a favor de los estudiantes del último año, solo cuando se utiliza el método de estimación MNLM. Por lo anterior, la hipótesis 5 resulta rechazada.

- Hipótesis 6: aquellos estudiantes que reciben formación emprendedora reconocen el esfuerzo por parte de la universidad en ese sentido.

En referencia a esta cuestión, se encuentra cierto apoyo para aceptar esta hipótesis. Los valores hallados a lo largo del análisis descriptivo (y con 
diferencias estadísticamente significativas) muestran que los estudiantes con educación emprendedora, además de considerar la opción de emprender como una alternativa profesional posible, reconocen que se les han dado herramientas útiles si en algún momento de sus vidas deciden hacerlo. Por otro lado, también reconocen haber afianzado su conocimiento sobre el mundo emprendedor, así como también haber extraído algunas recomendaciones sobre las buenas prácticas a la hora de emprender y haber desarrollado habilidades necesarias para ser un emprendedor. Asimismo, del análisis de regresión surge que la educación emprendedora tiene una relación positiva con la intención de emprender, lo que sumado a lo anterior se podría afirmar que es posible egresar potenciales emprendedores formados. En suma, estos resultados permiten aceptar la hipótesis 6 .

\section{Conclusiones}

A lo largo del presente trabajo se estudió la intención emprendedora en estudiantes universitarios de diferentes áreas del conocimiento, y con diferente formación en lo referido a haber recibido o no educación emprendedora dentro de sus planes de estudio. El objetivo era el de evaluar el efecto de cada tipo de formación en la decisión de su futuro profesional, en lo que hace a la posibilidad de comenzar con su propio emprendimiento.

Para ello, se analizaron previamente ciertas particularidades en los estudiantes que tienen relación con la temática emprendedora, entre las que se destaca que no hay gran presencia de madres y padres emprendedores, aunque en aquellos casos donde los hay, la valoración positiva está dada más respecto de los padres que de las madres.

Asimismo, cuando se analiza la percepción que presentan los estudiantes sobre lo que les ofrece el ámbito universitario respecto de la cuestión emprendedora, es notable el reconocimiento dado por los estudiantes pertenecientes a las tecnicaturas emprendedoras a la formación recibida, en lo que hace a las herramientas recibidas en el caso de que decidiesen emprender, el desarrollo de competencias, la trasmisión de buenas prácticas, entre otras, no siendo así en el resto de las carreras. Esto representa un antecedente 
importante en el caso de que se instale un debate sobre la inclusión o no de contenido emprendedor dentro de los actuales planes de carrera.

Otra cuestión de interés que es posible observar a lo largo del análisis es que, de acuerdo con las estimaciones realizadas, introducir un programa integral de educación emprendedora genera resultados positivos en su intención de emprender. Si bien la intención no es suficiente para dar lugar a una acción, ya que puede haber varios obstáculos en el camino (Carsrud, Brännback, 2011), lo que resulta de interés es que dicha posibilidad surja como una alternativa profesional posible. Esta cuestión se ve reflejada de manera contraria en el resto de las carreras donde pareciera que la inclinación sea más que nada hacia la búsqueda de empleo, producto de que quizás la universidad, no les haya brindado herramientas para autoemplearse.

\section{Referencias bibliográficas}

Ajzen, I. (1987). Attitudes, traits, and actions: Dispositional prediction of behaviour in social psychology. Advances in Experimental Social Psychology, n. ${ }^{\circ}$ 20, pp. 1-63. DOI: http:/ /dx.doi.org/10.1016/S0065-2601(08)60411-6

Ascúa, R., Andrés, M. F. y Danko, B. (2014). Señales de entrepreneurship entre los estudiantes universitarios argentinos. En Ruda, W., Ascúa, R., Danko, B. y Martin, T. A. (eds.). Propensión emprendedora de estudiantes universitarios Estudio GESt. Análisis y evaluación empírica en Europa y América Latina. Santa Fe: Ediciones UNL.

Braidot, N. (2001). Educación para la Empresarialidad en el contexto universitario Argentino: ¿Opción o Necesidad? Universidad Argentina de la Empresa (mimeograph).

Carsrud, A. \& Brännback M. (2011). Entrepreneurial Motivations: What Do We Still Need to Know? Journal of Small Business Management, n. ${ }^{\circ}$ 49(1), pp. 9-26.

Cesar, R., Fardelli Corropolese, C., Federico, J. y Baruj, G. (s.f.). Mapa de Instituciones Argentinas de Desarrollo Emprendedor. Instituto de industria. Universidad de General Sarmiento. Recuperado el 10 de agosto de 2010 de http://www.littec.ungs.edu.ar/e-books/mapa\%20instituciones.pdf

Cutidiano, R., Cesar, R., Porras, J. y Partal, C. (2006, septiembre). La educación emprendedora en el contexto universitario: la generación de emprendedores 
Analizando la influencia de la educación universitaria en el perfil emprendedor...

como objetivo institucional. En $11^{a}$ Reunión Anual Red Pymes Mercosur, realizadas en la Universidad Nacional del Centro, Tandil.

Fayolle, A. y Degeorge, J. M. (2006). Attitudes, intentions, and behaviour: New approaches to evaluating entrepreneurship education. En Fayolle, A., Klandt, H. (eds). International entrepreneurship education. Issues and newness. Cheltenham, UK and Northampton, MA, USA: Edward Elgar.

González López, M. J., García Piñeiro S. y Rodríguez Ariza, L. (2012, junio). Indicadores para la evaluación de la educación superior en emprendimiento: Una aproximación. En Workshop en Experiencias y Metodologías Docentes en Emprendimiento. Granada: actas del congreso.

Gorman G., Hanlon D. \& King W. (1997). Some Research Perspectives on Entrepreneurship Education. Enterprise Education and Education for Small Business Management: a ten-year Literature Review. International Small Business Journal, n. ${ }^{\circ}$ 15(3), pp. 56-77.

Guerrero, M., Rialp, J. \& Urbano, D. (2008). The Impact of Desirability and Feasibility on Entrepreneurial Intentions: a Structural Equation Model. International Entrepreneurship and Management Journal, n. ${ }^{\circ}$ 4(1), pp. 35-50.

Krueger N. F. \& Brazeal D. (1994). Entrepreneurial potential and potential entrepreneurs. Entrepreneurship Theory and Practice, n. ${ }^{\circ} 18$, pp. 91-104.

Liñán, F. (2004a). Educación empresarial y modelo de intenciones. Formación para un empresariado de calidad. Análisis empírico para la provincia de Sevilla (Tesis Doctoral). Universidad de Sevilla, Sevilla.

Liñán, F. (2004b). Intention-based models of entrepreneurship education. International. Small Business, n. ${ }^{\circ}$ 3, pp. 11-35.

Liñán, F., Rodríguez-Cohard, J. C. \& Rueda-Cantuche, J. M. (2011). Factors affecting entrepreneurial intention levels: a role for education. International Entrepreneurship and Management Journal, n. ${ }^{\circ}$ 7(2), pp. 195-218.

Martin, B. C., McNally, J. J. y Kay, M. J. (2013). Examining the formation of human capital in entrepreneurship: a meta-analysis of entrepreneurship education outcomes. Journal of Business Venturing, n. ${ }^{\circ} 28(2)$, pp. 211-224. DOI: http:// dx.doi.org/10.1016/j.jbusvent.2012.03.002

McMullan, W. E. D. \& Long, W. A. (1987). Entrepreneurship Education in the nineties. Journal of Business Venturing, n. ${ }^{\circ} 2$, pp. 261-275. 
Neck, H. M. \& Greene, P. G. (2011). Entrepreneurship education: known worlds and new frontiers. Journal of Small Business Management, n. ${ }^{\circ}$ 49(1), pp. 55-70. DOI: http://dx.doi.org/10.1111/j.1540-627X.2010.00314.x

Pittaway, L. \& Cope, J. (2007). Entrepreneurship education a systematic review of the evidence. International Small Business Journal, n. ${ }^{\circ}$ 25(5), pp. 479-510.

Porras, J., Partal, C., Amadío, C., Murello, D. y Cesar, R. (2006, septiembre). El desarrollo de capacidades emprendedoras a través de la educación primaria, secundaria y polimodal. En $11^{a}$ Reunión Anual Red Pymes Mercosur, realizada en la Universidad Nacional del Centro, Mendoza.

Porras, E., Oliveras, G., Vigier, H., Savoretti, A., Dichiara, R. y Bruno, M. (2009, diciembre). La formación de emprendedores y el fomento a la iniciativa empresarial: la experiencia de la Universidad Provincial del Sudoeste. En $I^{0}$ Jornada sobre PYME e Iniciativa Empresarial, realizada en la Universidad Carlos III, Getafe, Madrid.

Postigo, S. \& Tamborini, M. F. (2002, julio). Entrepreneurship education in Argentina: The case of San Andrés University. En Internationalizing Entrepreneurship Education and Training Conference, (IntEnt02), realizada en Malaysia.

Reyes Rodríguez, L. (2007). La teoría de acción razonada: implicaciones para el estudio de las actitudes. Investigación Educativa, n. ${ }^{\circ}$ 7, pp. 66-77.

Sampieri Hernández, R., Collado Fernández, C. y Lucio Baptista, P. (2010). Metodología de la investigación. (5.a. ed.). México, D. F.: McGraw-Hill Interamericana.

Shapero, A. (1982). The Social Dimensions of Entrepreneurship. En Kent, C., Sexton, D. \& Vesper, K. (eds.). Encyclopedia of entrepreneurship, pp. 72-90. Englewood Cliffs, NY: Prentice Hall.

Shiersmann, S., Graña F. y Liseras, N. (2002, septiembre). Vocación emprendedora en alumnos universitarios: el caso de la Facultad de Ciencias Económicas de la UNMDP y FASTA. En $7^{\circ}$ Reunión Anual de la Red PyMEs Mercosur, realizada en Rafaela, Santa Fe. 
Ussman, A., Postigo, S. (2000). O Papel da Universidade no Fomento da Funçao Empresarial. Anais Universitarios. Ciencias Sociais e Humanas. Número especial 1990-2000, pp. 219-233.

Vigier, H., Partal, C., Savoretti, A. y Porras, J. A. (2006). Las universidades y el desarrollo local en el sudoeste bonaerense. En Rofman A. (comp.), Universidad y Desarrollo Local, Aprendizajes y Desafios, pp. 291-305. Buenos Aires: Prometeo Libros.

Vigier, H., Jiménez, J. J. y Oliveras G. (2011, noviembre). La incidencia de la educación universitaria en la intención emprendedora. Estudio comparativo en estudiantes universitarios de la Argentina. En II Jornadas de Iniciativas Empresariales, Pymes y Empresa Familiar, realizada en la Universidad de Pablo de Olavide, Sevilla.

Walter, S. G., Parboteeah, K. P. y Walter, A. (2013). University departments and self-employment intentions of business students: a cross-level analysis. Entrepreneurship Theory and Practice, n. ${ }^{\circ} 37(2)$, pp. 175-200. DOI: http://dx.doi. org/10.1111/j.1540-6520.2011.00460.x

Wilson, K. E., Vyakarnam, S., Volkmann, C., Mariotti, S. \& Rabuzzi, D. (2009, abril). Educating the next wave of entrepreneurs: Unlocking entrepreneurial capabilities to meet the global challenges of the 21st century. En World Economic Forum: A Report of the Global Education Initiative, Switzerland: World Economic Forum. 


\section{ANEXO}

\section{MODELO DE CUESTIONARIO UTILIZADO PARA EL RELEVAMIENTO EN ESTUDIANTES}

1. Edad:

2. Sexo:

\begin{tabular}{|l|l||l|l|}
\hline Femenino & & Masculino & \\
\hline
\end{tabular}

3. Año qué estas cursando:

\begin{tabular}{|l|l||l|l|}
\hline Primero & & Último & \\
\hline
\end{tabular}

4. ¿Qué carrera estás cursando?

5. Indicá tu grado de acuerdo con cada una de las afirmaciones que siguen, usando la siguiente escala: 1 = totalmente en desacuerdo, 2 = en desacuerdo, $3=$ parcialmente en desacuerdo, $4=$ indiferente/no lo he pensado, $5=$ parcialmente de acuerdo, 6 = de acuerdo, 7 = completamente de acuerdo.

En mi caso particular...

... tengo intención de tener mi propia empresa luego de graduarme.

... encuentro atractiva la idea de crear mi propia empresa.

... siento que me resultaría factible crear mi propia empresa.

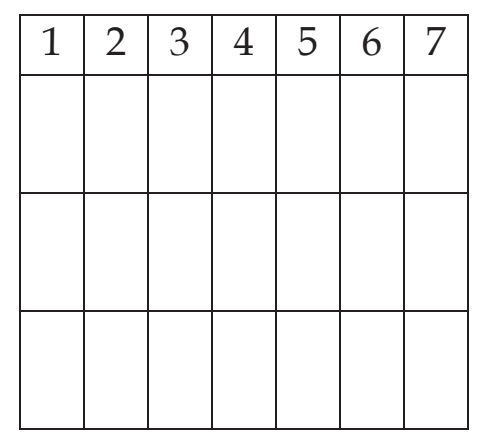

\section{Respecto a mi conocimiento emprendedor...}

... tengo en claro las políticas del Estado para financiar emprendimientos.

... tengo en claro la función y los aportes de las incubadoras de empresas.

... estoy al tanto de los programas disponibles para apoyo a los emprendedores.

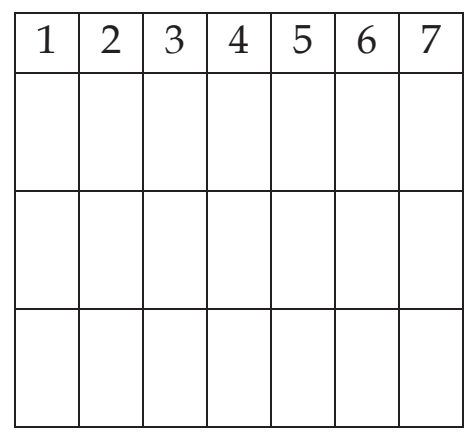


Analizando la influencia de la educación universitaria en el perfil emprendedor...

6. ¿Alguna de las siguientes personas de tu entorno está llevando a cabo su propio emprendimiento?

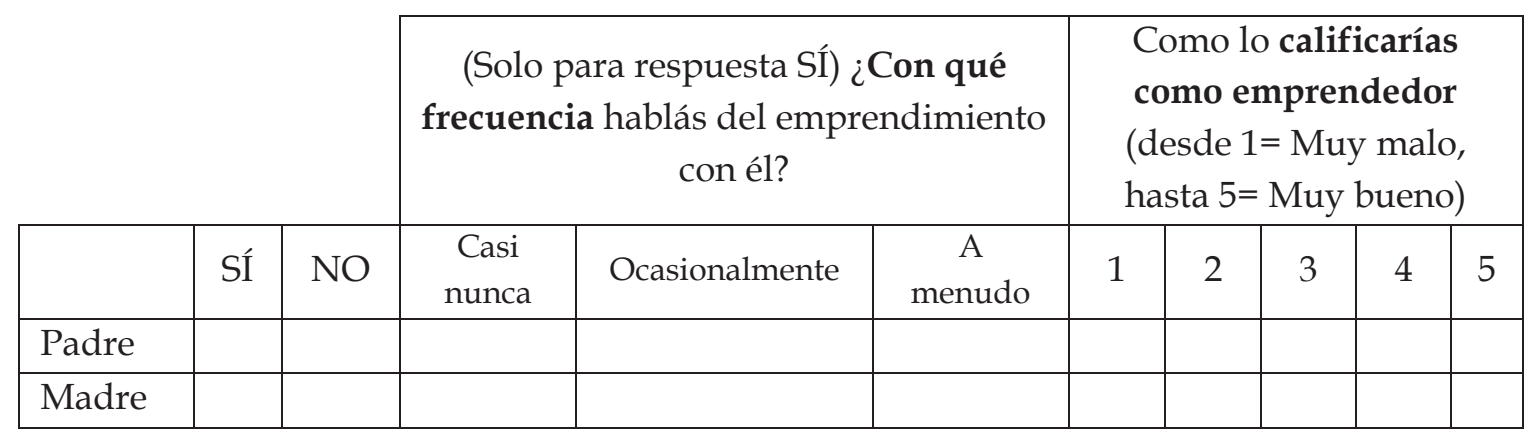

7. En lo que hace a una idea de negocios... (seleccioná con una X $\underline{\text { solo una opción) }}$

Hoy no tengo ninguna idea

Sí tengo una idea...

... estoy evaluando esa idea SÍ $\quad \mathrm{NO}$

8. Inmediatamente luego de finalizar tu carrera universitaria, ¿qué grado de preferencia tienen para vos las siguientes alternativas profesionales?

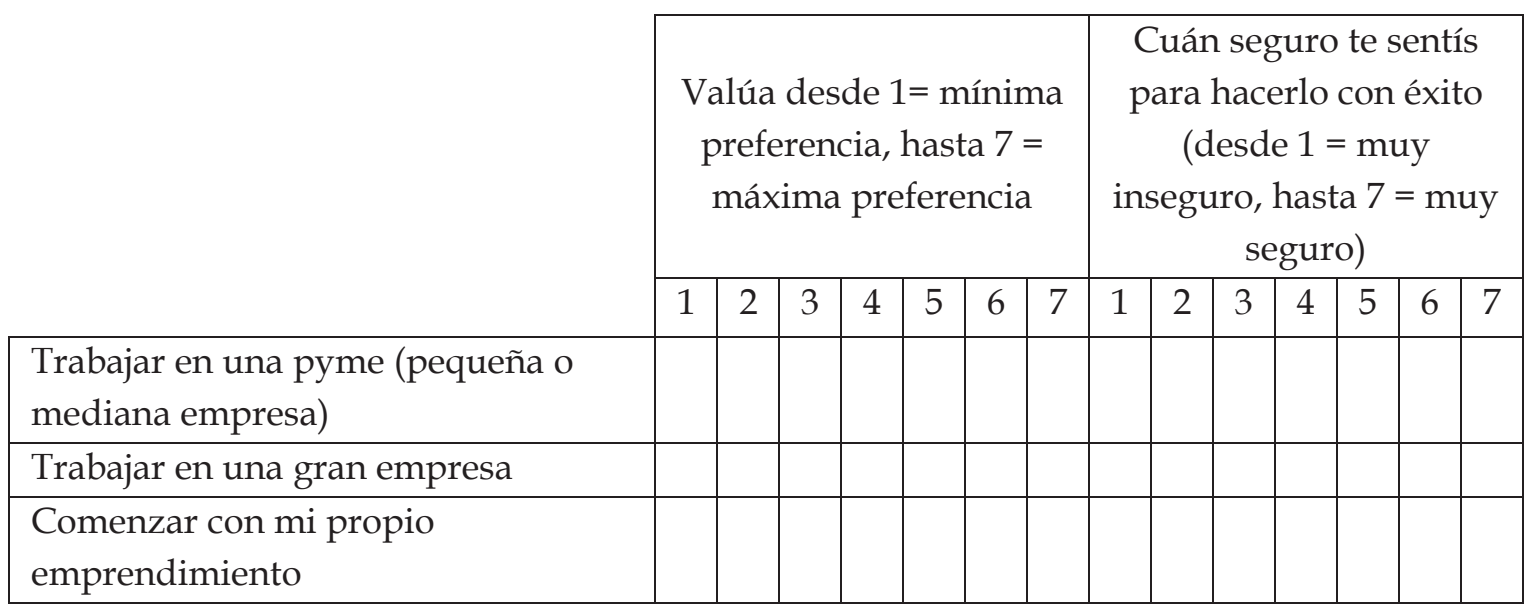




\section{A CONTINUACIÓN, SOLO RESPONDÉ SI SOS ALUMNO DEL ÚLTIMO AÑO DE TU CARRERA UNIVERSITARIA}

9. Respecto de tu educación universitaria, indicá tu grado de acuerdo con las siguientes afirmaciones... (desde $1=$ totalmente en desacuerdo hasta $7=$ completamente de acuerdo).

\begin{tabular}{|c|c|c|c|c|c|c|c|}
\hline & 1 & 2 & 3 & 4 & 5 & 6 & 7 \\
\hline $\begin{array}{l}\text { Durante mi carrera he adquirido herramientas técnicas, útiles } \\
\text { si en algún momento de mi vida deseo crear mi propia } \\
\text { empresa }\end{array}$ & & & & & & & \\
\hline $\begin{array}{l}\text { Siento que me ha preparado más para trabajar en una gran } \\
\text { empresa que para crear mi emprendimiento }\end{array}$ & & & & & & & \\
\hline $\begin{array}{l}\text { Me ha dejado en claro que tener mi propio emprendimiento } \\
\text { puede ser una alternativa profesional posible }\end{array}$ & & & & & & & \\
\hline $\begin{array}{l}\text { Durante mi carrera he afianzado mi conocimiento sobre el } \\
\text { mundo emprendedor }\end{array}$ & & & & & & & \\
\hline $\begin{array}{l}\text { He generado buenos vínculos, que serán útiles en caso de } \\
\text { querer llevar a cabo mi emprendimiento }\end{array}$ & & & & & & & \\
\hline $\begin{array}{l}\text { Pude extraer algunas recomendaciones generales sobre las } \\
\text { buenas prácticas a la hora de emprender, que me serán útiles si } \\
\text { en algún momento de mi vida deseo hacerlo }\end{array}$ & & & & & & & \\
\hline $\begin{array}{l}\text { Me ha permitido desarrollar habilidades necesarias para ser un } \\
\text { emprendedor }\end{array}$ & & & & & & & \\
\hline $\begin{array}{l}\text { Algún docente me transfirió conocimientos y experiencias en } \\
\text { el tema emprendedor }\end{array}$ & & & & & & & \\
\hline
\end{tabular}


Analizando la influencia de la educación universitaria en el perfil emprendedor...

${ }^{1}$ Este artículo forma parte del trabajo de investigación realizado por Guadalupe Oliveras, para acceder al título de Doctora en Ciencias de la Investigación, bajo la dirección del Dr. Hernán P. Vigier y el Ing. Qco. José A. Porras.

2 Doctora en Ciencias de la Administración. Profesora Adjunta en la Universidad Provincial del Sudoeste. Auxiliar de docencia en el Departamento de Ciencias de la Administración de la Universidad Nacional el Sur. guadalupe.oliveras@upso.edu.ar

${ }^{3}$ Doctor en Administración y Dirección de Empresas. Profesor Titular en el Departamento Economía de la Universidad Nacional del Sur. Profesor Titular en la Universidad Provincial del Sudoeste.hvigier@upso.edu.ar

4 Ingeniero Químico. Director del Centro de Emprendedorismo y Desarrollo Territorial Sostenible (CIC-UPSO). Profesor Titular en el Departamento de Ingeniería Química de la Universidad Nacional del Sur. Profesor Titular en la Universidad Provincial del Sudoeste. jporras@upso.edu.ar

${ }_{5}^{5}$ Se considera que esta característica resulta del hecho de que este tipo de estudiantes provienen de poblaciones que por primera vez pueden acceder a una enseñanza universitaria a través de la llegada de las tecnicaturas, y, por lo tanto, muchos estudiantes son personas de mayor edad que pudieron tener acceso a estudios universitarios recién en ese entonces.

${ }^{6}$ En la provincia de Santa Fe, no se verifica relación de efecto significativa en los estudiantes según sea la especialidad de estudios.

7 Salvo en estudiantes del área de Ciencias Biológicas. 